\title{
Prevalence of mycobacterium tuberculosis and their possible risk factors in suspected tuberculosis patients of district mardan khyber pakhtunkhwa, Pakistan
}

\begin{abstract}
Tuberculosis (TB) is one of the chronic contagious diseases caused by Mycobacterium Tuberculosis that affect humans and animals. It is stated that TB is one of the major leading cause of morbidity and mortality throughout the globe. According to the WHO, approximately one-third people of the world are infected and about 3 million people die annually due to this disease. The present study reported the incidence rate of pulmonary $\mathrm{TB}$ and their possible risk factors in suspected TB patients according to age, gender, and location-wise prevalence of the disease in District Mardan, Khyber Pakhtunkhwa, Pakistan. A total of 380 sputum samples were collected from infected Tuberculosis patients and initially screened for acid-fast bacilli through Zeihl Neelson (ZN) microscopy and positive samples were further assayed by Real-time Polymerase Chain Reaction (GeneXpert). Out of these sputum samples $40 \%$ were positive for $\mathrm{ZN}$ staining and $31.57 \%$ were positive by GeneXpert. The prevalence rate was reported high in the age group of above sixty while a lower prevalence rate was found in the age group of 5-20 years. Male to female ratio was recorded at $37.83 \%$ and $41.37 \%$ respectively. Geographically, rural populations were highly infected by $M$. Tuberculosis which showed $45.19 \%$ as compared to the urban population showed $26.16 \%$ prevalence rate. All the relative risk factors are statistically significant having $\mathrm{p}$-value $(<0.05)$. It was concluded from our finding that $\mathrm{TB}$ was considerably increased in the general population of District Mardan, and proper supervision, diagnosis, treatment, and awareness of rapid prophylactic measures are needed to eradicate the risk.
\end{abstract}

Volume 6 Issue 4 - 2019

\author{
Muhammad Naveed, 1,2 Muhammad Shahid, ${ }^{2}$ \\ Adil Hassan, ${ }^{3}$ Mujeeb ur Rahman, ${ }^{4}$ Azam \\ Hayat $^{5}$ \\ Institute of Microbiology, China \\ ${ }^{2}$ Department of Microbiology, Pakistan \\ ${ }^{3}$ Chongqing University, China \\ ${ }^{4}$ Northwest University, China \\ ${ }^{5}$ Department of Microbiology, Pakistan
}

\begin{abstract}
Correspondence: Muhammad Naveed, Institute of Microbiology, School of Life Sciences, Lanzhou University, Lanzhou 730000, People's Republic of China, Tel +008613038727754, Email naved20I7@|zu.edu.cn
\end{abstract}

Received: November 15, 2019 | Published: November 25, 2019

Keywords: tuberculosis, zeihl neelson staining, PCR, genexpert

Abbreviations: TB, tuberculosis; ZN, zeihl neelson; PCR, polymerase chain reaction; DM, diabetes mellitus; WHO, world health organization

\section{Introduction}

Tuberculosis (TB) is one of the chronic contagious diseases caused by M.tuberculosis that affects humans and animals and one of the leading causes of morbidity and mortality throughout the globe., ${ }^{1,2}$ It primarily causes pulmonary tuberculosis by affecting lungs of the patients but can also infects bones, meninges, joints, intestines, skin, lymph glands, kidneys, and body's further organs during its second phase of infection and cause extra pulmonary tuberculosis. ${ }^{3}$ At the initial phase of infection, most of the individuals show symptomless tuberculosis while most of the patients developed imprecise symptoms like mild cough, wheeze, weight loss, evening pyrexia, night sweats, increased weight loss, lethargy, and general ill-health. However specific symptoms may be determined by the site of infection. ${ }^{4} M$. tuberculosis is frequently spread from infected tuberculosis patients to other persons by aerosolized droplet nuclei which are generated during coughing, speaking, laughing sneezing and shouting. The droplets nuclei may persist in the air for some time and dry quickly, and when these nuclei breathe in by the individual it can achieve direct entrance to the terminal air passages. The infected person may be producing more than three thousand contagious particles per cough. ${ }^{5}$ It concerns a major health issue and cause serious illness among millions of people annually and considered the second leading cause of death after HIV infection. ${ }^{2}$

Several studies showed that lack of education, non-availability of qualified staff, insufficient medical coverage, malnourished individuals, people suffered from HIV/AIDS, people infected with cancer. Those living with somebody who has active tuberculosis, health care labors in hospitals are in high risks to get tuberculosis. ${ }^{6,7}$ Several scientists demonstrated that tobacco smoking and TB has strongly correlated and considered as the main threat to the development of tuberculosis ${ }^{8,9}$ Indirect smoking was also contributed to being a major risk factor, and control of smoking could have a significant impact on the incidence and prevalence rate of tuberculosis. ${ }^{10}$ Approximately, 1.3 billion citizens in underdeveloped or developing countries use tobacco and lead to a higher tuberculosis rate throughout the world. ${ }^{11}$ Diabetes mellitus (DM) was also considered the key factor for the development of tuberculosis infection from latent to the active stage and about $15 \%$ of tuberculosis cases might be associated with diabetes mellitus globally. ${ }^{12,13}$ In 2014, World Health Organization (WHO) reported that one-third of the global population was infected with tuberculosis and data collected from 2013 showed that approximately 9 million new cases of positive tuberculosis were reported worldwide. ${ }^{14}$ Tuberculosis has been prevalent in Pakistan and contributed $44 \%$ of the TB disease globally. ${ }^{15}$ Pakistan is ranked $8^{\text {th }}$ in the prevalence of 
TB with an estimate of 1.5 million tuberculosis patients. ${ }^{16}$ Aim of the current study was to find out the prevalence of tuberculosis and their associated risk factors in suspected TB patients of district Mardan Khyber Pakhtunkhwa, Pakistan.

\section{Material and methods}

\section{Study area}

The current study was conducted at the regional tuberculosis Control Program, Mardan Medical complex \& Teaching hospital (MMC\&TH) Mardan, Khyber PakhtunKhwa, Pakistan. Sputum samples were randomly collected from the tuberculosis-infected patients along with the history in the main laboratory of Mardan Medical Complex \& Teaching Hospital Mardan KpK, Pakistan.

\section{Sample size}

A total of 380 Participants were included in the current study. Participants belonged to other districts than district Mardan and having age limit less than 05 years were excluded from this study.

\section{Methodology}

Sputum samples were collected in sterile plastic bottles in triplicate from all the enrolled participants and stored at $4{ }^{\circ} \mathrm{C}$ in the laboratory. A complete informative and designed questionnaire was used for the detailed history of each patient and to report the potential threat features for the infection of M. tuberculosis. All collected samples were liquefied and decontaminated using a standard procedure using sodium hydroxide and isopropanol for the digestion and decontamination of sputum specimens and processed through Zeihl Neelsen staining microscopy and real-time PCR (Gene Xpert).

\section{Zeihl neelsen (ZN) staining microscopy for the detection of acid fast bacilli}

Sputum smear was prepared by spreading the sputum on a glass slide and fix it with a flame. Covered the smear with sufficient carbolfuchsin stain and heat it with the help of Bunsen burner until to evaporate and leave the slide for 5 minutes. The smear was completely decolorized with $3 \%$ acid-alcohol for five minutes that become visible to the naked eye. Washed the smear carefully with clean water, drained the slide and covered with the counter-stain (Methylene Blue) for 60120 seconds. Rinsed the smear with clean water, allowed to dry and examined the smear microscopically, using the 100x oil immersion objective. ${ }^{17}$ Following staining, identification of AFB was performed under microscope as per the WHO guideline.

\section{GeneXpert approach for the detection of $M$ tuberculosis}

In this method, Sputum samples were treated with sodium hydroxide and isopropanol buffer with the ratio of $(2: 1)$ and incubated it for 5 minutes at room temperature for complete detoxification and liquefaction. After incubation, $2 \mathrm{ml}$ of the eluted sample was transferred into the Cepheid cartridge that contains the wash buffer, reagents for lyophilized DNA extraction and PCR amplification. The reaction cartridge was put in the GeneXpert instrument for filtering, extraction of DNA and PCR amplification. MTB organisms from the sputum sample were first captured on the membrane filter by GeneXpert and washed the inhibitor from the captured cell with the washing buffer. Through sonication, the captured cells were lysed and nucleic acid (DNA) was released which was mixed with dried-down bead reagents in the PCR tube, where PCR amplification and detection occurred simultaneously. Specific oligonucleotide probes were hybridized with PCR products and six color bands were developed with a hybridization reaction. The overall flow of the GeneXpert reaction is shown in (Figure 1).

\section{Lay-out of GeneXpert assay}
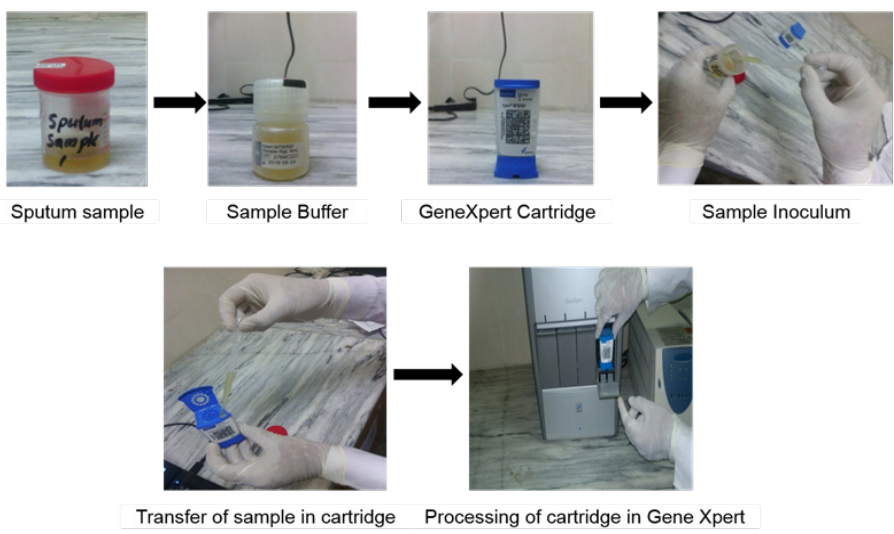

Figures I Overall flow of the sputum sample processing at Cepheid GeneXpert Machine.

\section{Statistical analysis}

All the data collected from the above mention assays were in a computer and statistically analysis was carried out by Graph Pad prism version 5. Percentages and ratios were calculated for different variables. A P-value $(<.05)$ are considered statistically significant.

\section{Results}

A total of 380 samples were received from the infected tuberculosis patients in which 152 samples $(40 \%)$ were screened positive for tuberculosis by $\mathrm{ZN}$ staining while, 120 samples (31.57\%) were positive by GeneXpert as shown in (Figure 2).

Detection of M.tuberculosis by ZN staining and GeneXpert

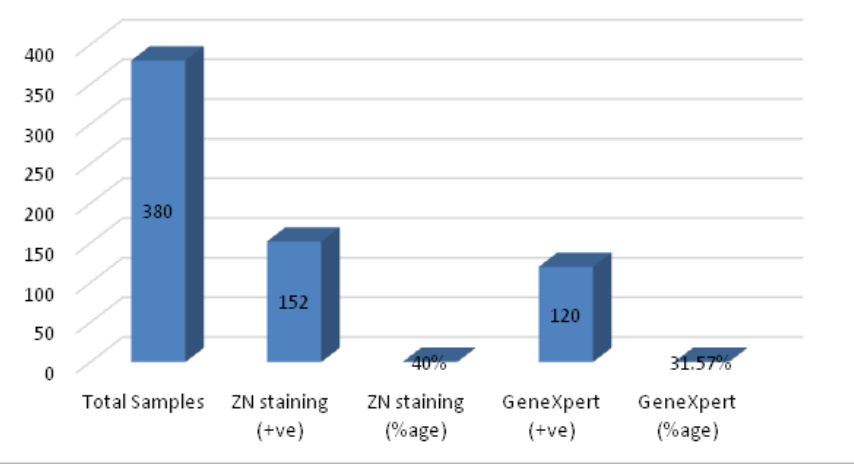

Figure 2 Detection rate of $M$. tuberculosis by ZN staining and GeneXper.

\section{Age wise distribution of $M$. tuberculosis prevalence in} district Mardan

In the current study a total 380 samples were registered in which the high number of positive cases were reported in the age group of $>60$ years which were $64.28 \%$ of the total samples, while $39.15 \%$ cases were found in the age group of 21 to 40 years which was that 
second highest prevalence rate in this study. Furthermore, $37.28 \%$ of cases were screened positive in the age group of 41 to 60 years and it was $36.76 \%$ for the age group of 5 to 20 years as shown in (Table 1).

Sex wise distribution of $M$. tuberculosis prevalence in district Mardan

Of the total cases $152(40 \%)$ were positive comprises male to female ratio was $56(37.83 \%)$ and was $96(41.37 \%)$ respectively by ZN staining, while male to female ratio was $42(28.37 \%)$ and 78 $(33.62 \%)$ by GeneXpert analysis as shown in (Table 2 ).

Table I Age wise distribution of $M$. tuberculosis prevalence in district Mardan

\section{Geographic distribution of $M$. tuberculosis prevalence in district Mardan}

On the basis of geographic distribution of M. tuberculosis prevalence in district Mardan 94 (45.19\%) cases were reported positive from rural population by ZN staining and $85(40.86 \%)$ by GeneXpert while from the urban population those cases were $45(26.16 \%)$ by $\mathrm{ZN}$ staining and 35(20.34\%) by GeneXpert as shown in (Table 3 ).

\begin{tabular}{llllll}
\hline Age group & Samples & ZN staining (+ve) & ZN staining (\%age) & GeneXpert (+ve) & GeneXpert (\%age) \\
\hline $20-$ May & 68 & 25 & $36.76 \%$ & 22 & $32.35 \%$ \\
$21-40$ & 166 & 65 & $39.15 \%$ & 40 & $24 \%$ \\
$41-60$ & 118 & 44 & $37.28 \%$ & 45 & $38.13 \%$ \\
$>60$ & 28 & 18 & $64.28 \%$ & 13 & $46.42 \%$ \\
Total & 380 & 152 & $40 \%$ & 120 & $31.57 \%$ \\
\hline
\end{tabular}

Table 2 Sex wise distribution of M. tuberculosis prevalence in district Mardan

\begin{tabular}{llllll}
\hline Sex & Samples & ZN staining (+ve) & ZN staining (\%age) & GeneXpert +ve & GeneXpert (\%age) \\
\hline Male & 148 & 56 & $37.83 \%$ & 42 & $28.37 \%$ \\
Female & 232 & 96 & $41.37 \%$ & 78 & $33.62 \%$ \\
Total & 380 & 152 & $40 \%$ & 120 & $31.57 \%$
\end{tabular}

Table 3 Geographic distribution of $M$. tuberculosis prevalence in district Mardan

\begin{tabular}{llllll}
\hline Area & Samples & ZN staining (+ve) & ZN staining (\%age) & GeneXpert (+ve) & GeneXpert (\%age) \\
\hline Rural & 208 & 94 & $45.19 \%$ & 85 & $40.86 \%$ \\
Urban & 172 & 45 & $26.16 \%$ & 35 & $20.34 \%$ \\
Total & 380 & 152 & $40 \%$ & 120 & $31.57 \%$ \\
\hline
\end{tabular}

\section{Distributions of M.tuberculosis Individuals in risk group}

Here (Table 4) summarized the Distributions of M.tuberculosis Individuals in their relative risk Groups. The maximum percentage of $25 \%$ was observed in individuals having previous history of antiTB medication which is statistically non-significant $(\mathrm{P}=0.3043)$.
Similarly, other risk group includes history of smoking (14.47\%) $(\mathrm{P}=0.0272)$, history of diabetes $(8.55 \%) \quad(\mathrm{P}=0.004)$, history of hepatitis $(3.94 \%)(\mathrm{P}=0.0411)$ and previous history of contact with MTB positive patients which were $7.89 \%(\mathrm{P}=0.0035)$. All the results are statistically significant and p-value less than .05 .

Table 4 Distributions of $M$. tuberculosis individuals in risk group

\begin{tabular}{llllll}
\hline S. No & Possible risk factors & +ve Cases & $\%$ age & P-value & R. Risk \\
\hline 1 & History of Smoking & 22 & $14.47 \%$ & 0.0272 & 1.480 \\
2 & History of Diabetes & 13 & $8.55 \%$ & 0.004 & $1.88 \mathrm{I}$ \\
3 & History of Hepatitis & 6 & $3.94 \%$ & 0.0411 & 1.911 \\
4 & History of previous anti-TB medication & 38 & $25 \%$ & 0.3043 & 0.8616 \\
5 & History of Contact with MTB positive patients & 12 & $7.89 \%$ & 0.0035 & 1.950 \\
& Total & 152 & $100 \%$ & & \\
\hline
\end{tabular}




\section{Discussion}

Tuberculosis is one of the most contagious disease infected those people who live in crowded, ill-ventilated places and those who are ill-fitted to conflict tuberculosis in term of their immune status or whose nutritious level is so low that they easily capitulated to tuberculosis. ${ }^{18}$ According to the Global TB Report in 2008, World Health Organization revealed that Pakistan is on 8th positions among the twenty-two high-burden tuberculosis countries worldwide, where approximately 280.000 people develop TB primarily adults in their creative years. ${ }^{18}$ Similar to other growing countries, the high risk groups for tuberculosis infection in Pakistan, may comprise individuals with diabetes, people with hepatitis, HIV/AIDS infected people or people suffered from cancer, those individuals who have been contacting with active tuberculosis patients, residents of homeless shelters, the malnourished, poor and impecunious people and present or former prisoners. ${ }^{12,19}$ According to the WHO recommendation, a new molecular technique, GeneXpert has been used for the rapid detection of $M$. tuberculosis and can be used to screen those strains which show resistance to Rifampicin mainly in extra pulmonary samples. ${ }^{20}$ The result of GeneXpert shows a determined positivity rate which stated that it is an absolute specific and sensitive technique as compared to conventional methods.

In the present study, $152(40 \%)$ out of 380 collected sputum specimens were observed positive under Ziehl Neelsen microscopy by oil immersion lens which showed pink, rod-shaped Mycobacterium with a blue background. Similarly, other studies showed $10.6 \%$ and $20.0 \%$ prevalence rate for acid-fast bacilli by the Zeihl Neelson technique. ${ }^{21,22}$ The results showed that the prevalence of M.tuberculosis was high in the age groups of $>60$ which were $(64.28 \%)$. Similarly, in the age group of $21-40,(39.15 \%)$ and $41-60,(37.28 \%)$ while a low prevalence rate was recorded between the age groups 5-20 years which was $36.76 \%$. This result shows that age affects the prevalence of tuberculosis.

The higher prevalence rate in the age groups of greater than 60 years may be attributed to the greater contact to the risk factors and prolong tuberculosis infection. Several researchers showed that tuberculosis is more prevalent in old stages mostly in old men due to their suppressive immune system using various drugs, smoking, and alcohol which may lead to disease development at a high rate. ${ }^{23,24}$

The maximum percentage of $41.37 \%$ was reported in female patients while $37.83 \%$ of patients were recorded in male. Our study results are similar to other studies that reported a high rate of tuberculosis prevalence in the female population as compared to male. ${ }^{25}$ Females are more susceptible to disease development as compare to males because of extra immune deficient and some hormonal changes in their reproductive years which may lead to at a higher level of disease progression. ${ }^{26}$ In Hong Kong study reported the highest rate of tuberculosis prevalence in women community observed mostly in their reproductive ages and due to missing of anti-TB medication. ${ }^{23}$ Geographically, the present study shows the highest prevalence rate of TB in the rural population of district Mardan which is $45.19 \%$ while this rate of prevalence is lower in the urban community which is $26.16 \%$. Mainly the rural areas suffered to poverty, poor diagnosis, non-availability of qualified staff, insufficient education rate, and unsatisfactory coverage of immunization.

The distributions of M. Tuberculosis Individuals in their relative risk groups are also discussed with respect to their statistical p-value. The maximum percentage of $25 \%$ was observed due to previous history of anti-TB medication ( $\mathrm{P}=0.3043)$ which is statistically nonsignificant. Similarly, other risk factor includes history of smoking $(14.47 \%)(\mathrm{P}=0.0272)$, history of diabetes $(8.55 \%)(\mathrm{P}=0.004)$, history of hepatitis $(3.94 \%)(\mathrm{P}=0.0411)$ and previous history of contact with MTB positive patients which were $7.89 \%(\mathrm{P}=0.0035)$. All the results are statistically significant and $p$-value less than 0.05 . The results of our study are correlated with other previous studies. ${ }^{12,19}$

\section{Conclusion}

The current study showed that most of the people living in rural area were infected by $M$. tuberculosis, and its percentage varies among different age groups, geographically and male to female community. The screening of $M$. tuberculosis in the local population of district Mardan showed high prevalence rate in female population as compared to male. Moreover, this ratio was also high in the age group of greater than sixty years while low prevalence rate was reported in the age group of 5-20 years. It is suggested to pay full attention on diagnosis and to treat the early phase of tuberculosis infection in Pakistan and to develop an efficient control program for the eradication of this disease.

\section{Funding}

None.

\section{Acknowledgments}

We are thankful to the department of pathology, Mardan Medical Complex \& Teaching Hospital Mardan (MMC\&TH) for providing us the required resources for the completion of the project.

\section{Conflicts of interest}

The authors of this manuscript do not have any conflict and competing of interest.

\section{References}

1. Sani R, Garba S, Oyeleke S, et al. Prevalence of pulmonary tuberculosis (PTB) in Minna and Suleja Niger state, Nigeria. American Journal of Medicine and Medical Sciences. 2015;5(6):287-91.

2. Alemu J, Mamo G, Kandi V, et al. Prevalence of Tuberculosis in Gambella Regional Hospital, Southwest Ethiopia: A Retrospective Study to Assess the Progress towards Millennium Development Goals for Tuberculosis (2006-2015). American Journal of Public Health. 2017;5(1):6-11.

3. Van Rie A, Enarson D. XDR tuberculosis: an indicator of public-health negligence. The Lancet. 2006;368(9547):1554-1556.

4. Tariq S, Rauf A, Malik S, et al. Presentations of tuberculosis in northern Pakistan. J Ayub Med Coll Abbottabad. 2011;23(1):40-42.

5. Cegielski JP, Chin DP, Espinal MA, et al. The global tuberculosis situation: progress and problems in the 20th century, prospects for the 21st century. Infect Dis Clin North Am. 2002;16(1):1-58.

6. Sharp J, Goldman M. Abdominal tuberculosis in East Birmingham--a 16 year study. Postgrad Med J. 1987;63(741):539-542.

7. Sonnenberg P, Glynn JR, Fielding K, et al. HIV and pulmonary tuberculosis: the impact goes beyond those infected with HIV. Aids. 2004;18(4):657-662.

8. Sahiratmadja E, Nagelkerke N. Smoking habit as a risk factor in tuberculosis: a case-control study. Universa Medicina. 2016;30(3):189196. 
9. Maurya V, Vijayan V, Shah A. Smoking and tuberculosis: an association overlooked. Int J Tuberc Lung Dis. 2002;6(11):942-951.

10. Leung CC, Lam TH, Ho KS, et al. Passive smoking and tuberculosis. Arch Intern Med. 2010;170(3):287-292.

11. van Zyl Smit R, Pai M, Yew WW, et al. Global lung health: the colliding epidemics of tuberculosis, tobacco smoking, HIV and COPD. Eur Respir J. 2010;35(1):27-33.

12. Silva DR, Muñoz-Torrico M, Duarte $R$, et al. Risk factors for tuberculosis: diabetes, smoking, alcohol use, and the use of other drugs. J Bras Pneumol. 2018;44(2):145-152.

13. Silva DR, Mello FCdQ, Kritski A, et al. Tuberculosis series. J Bras Pneumol. 2018;44(2):71-72.

14. Global tuberculosis report 2013. World Health Organization. 2013.

15. Vermund SH, Altaf A, Samo RN, et al. Tuberculosis in Pakistan: A decade of progress, a future of challenge. J Pak Med Assoc. 2009;59(4):1-8.

16. Global tuberculosis control: epidemiology strategy financing: WHO report 2009. World Health Organization. 2009.

17. Raviglione MC, Pio A. Evolution of WHO policies for tuberculosis control, 1948-2001. The Lancet. 2002;359(9308):775-780.

18. Manzoor S, Tahir Z, Anjum A. Prevalence Of Hiv And Tuberculosis Among Jail Inmates In Lahore-Pakistan. Medicine. 2009;25.

19. Dobler CC, Flack JR, Marks GB. Risk of tuberculosis among people with diabetes mellitus: an Australian nationwide cohort study. $B M J$ open. 2012;2(1):e000666.
20. Helb D, Jones M, Story E, Boehme C, Wallace E, Ho K, et al. Rapid detection of Mycobacterium tuberculosis and rifampin resistance by use of on-demand, near-patient technology. J Clin Microbiol. 2010;48(1):229-237.

21. Zailani S B, Lasan B, Danlami D. Detection Rates of Ziehl-nelseen Staining Technique and Fluoresecnt Microscopy in The Examination of Sputum for Acid Fast Bacilli. BOMJ. 2012;9(2):27-30.

22. Anita P, Madan M, Asthana AK, et al. Cold acid fast staining method: Efficacy in diagnosis of Mycobacterium tuberculosis. African Journal of Microbiology Research. 2009;3(9):546-549.

23. Chan-Yeung M, Noertjojo K, Chan S, et al. Sex differences in tuberculosis in Hong Kong. The Int J Tuberc Lung Dis. 2002;6(1):1118 .

24. Chan-Yeung M, Yeh A, Tam C, et al. Socio-demographic and geographic indicators and distribution of tuberculosis in Hong Kong: a spatial analysis. Int J Tuberc Lung Dis. 2005;9(12):1320-1326.

25. Uddin S, Khan FU, Ahmad T, et al. Two Years of Retrospective Study on the Incidence of Tuberculosis in Dir Lower Valley, Khyber Pakhtunkhwa, Pakistan. Hosp Pharm. 2018;53(5):344-349.

26. Cailhol J, Decludt B, Che D. Sociodemographic factors that contribute to the development of extrapulmonary tuberculosis were identified. $J$ Clin Epidemiol. 2005;58(10):1066-1071. 